\title{
Multifactorial modeling and optimization of solution and electrospinning parameters to generate superfine polystyrene nanofibers
}

\author{
Babak Rezaei $^{1}$ (D) | Ahmad Mousavi Shoushtari' ${ }^{2}$ (D) | Mohammad Rabiee ${ }^{3}$ | \\ Lokman Uzun ${ }^{4}$ | Anthony P. F. Turner ${ }^{4}$ | Wing Cheung Mak
}

${ }^{1}$ Nanotechnology Institute, Amirkabir University of Technology, Tehran, Iran

${ }^{2}$ Textile Engineering Department, AmirKabir University of Technology, Tehran, Iran

${ }^{3}$ Biomaterials Group, Biomedical Engineering Department, Amirkabir University of Technology, Tehran, Iran

${ }^{4}$ Department of Physics, Chemistry and Biology (IFM), Biosensors \& Bioelectronics Centre, Linköping University, Linköping, Sweden

\section{Correspondence}

Ahmad Mousavi Shoushtari, Textile Engineering Department, AmirKabir University of Technology, Tehran, Iran. Email: amousavi@aut.ac.ir

Funding information

Ministry of Science, Research and

Technology of Iran

\begin{abstract}
This study was conducted to provide a quantitative understanding of the influence of the different solution and electrospinning variables on the morphology and the mean diameter of electrospun polystyrene nanofibers. In this regard, the effect of different solvents and ionic additives on the electrical conductivity, viscosity, and surface tension of the electrospinning solutions and thereby the morphology of nanofibers were examined. The results indicated that the morphology of the fibers is extremely dependent on the solvent's properties, especially volatility and electrical conductivity, and the ionic characteristics of additives. Finally, to estimate the optimal electrospinning conditions for production of nanofibers with minimum possible diameter, modeling of the process was undertaken using the response surface methodology. Experimentally, nanofibers with the finest diameter of $169 \pm 21 \mathrm{~nm}$ were obtained under the optimized conditions, and these could be considered promising candidates for a wide practical range of applications ranging from biosensors to filtration.
\end{abstract}

K E Y W O R D S

electrospinning process, fibers, ionic additives, modeling, polystyrene

\section{1 | INTRODUCTION}

Owing to the outstanding advantages of nanofibers, such as their very large surface-area-to-volume ratio, high surface free energy, high porosity and interconnectivities, low barrier to diffusion of materials, and structural mechanical properties, these nanostructured materials have become more and more alluring for many practical applications. ${ }^{[1]}$ Although several methods have been devised in recent decades to generate nanofibers, including self-assembly, phase separation, and template synthesis, up to now, only electrospinning has been generally accepted and widely used. Electrospinning is an interesting and efficient process capable of producing continuous fibers from polymeric and ceramic materials with diameters in the range of nanometers to micrometers. ${ }^{[2]}$ In this process, usually a continuous strand of a polymer flow (i.e., solution or molten) is drawn through a spinneret by applying a high-voltage electric field, then nanofibers are randomly deposited onto a grounded metallic collector. ${ }^{[3]}$ Besides the above-mentioned merits of nanofibers, the nanofibers produced through the electrospinning process possess higher morphological uniformity and defect-free structure, owing to the nature of process, which is more controllable than the other methods mentioned. ${ }^{[4]}$

Numerous parameters influence the electrospinning process, and these can be classified into the following categories: solution, processing, and ambient parameters. ${ }^{[3]}$ Among them, the solution and processing parameters are the most crucial factors which should be considered to achieve fibers with favorable structural properties. The physical properties of solvent, such as volatility, electrical conductivity, and surface tension, can significantly influence the rheological and 
electrical properties of electrospinning solutions. Once the solvent volatility is high, solidification of the jet will take place in a very short time, which confines the jet stretching and elongation in the submicron fibers. Moreover, the high surface tension can also act against the stretching and elongation of the jet and disturb the formation of the desired fibers. ${ }^{[5]}$ To better understand the effect of the solutions' electrical conductivity on the electrospun nanofiber's morphology and diameter, a theoretical description of electrospinning process should be taken into consideration. In accordance with the principles of the electrospinning process, the electrically charged jet of the polymer solution experiences bending and whipping instabilities under the influence of an electrostatic field. ${ }^{[6]}$ It has been clearly demonstrated that these instabilities can cause stretching of the jet at very high frequencies and reduce the jet diameter. ${ }^{[7]}$ The instabilities are mainly dependent on the electric charge density on the surface of jet and the surface tension of the electrospinning solution. ${ }^{[6]}$ The surface tension tends to stabilize the jet and minimize its surface, while the electric charges on the surface tend to perturb and destabilize the jet. Ergo, it can be stated that a polymer solution with higher electrical conductivity could lead to thinner electrospun fibers, arising from the extension of bending and whipping instabilities. ${ }^{[8]}$

Polystyrene (PS) is one of the most interesting polymers to fabricate electrospun nanofibers due to its ability to dissolve in a large number of organic solvents, chemical inertness, physical-mechanical properties, excellent shape stability, and negligible swelling in aqueous media. ${ }^{[9]}$ It has been reported that electrospun PS nanofibers are very promising candidates for many practical applications such as biosensors, composite materials, enzyme immobilization, tissue engineering, filtration, ion exchangers, catalyst immobilization, and oil spill remediation. ${ }^{[10,11]}$ It is worth mentioning that having bead-free fibers with nanoscale diameter, smooth surfaces, and uniform structure (i.e., low variation in the morphology of nanofibrous mat) is crucial to achieving reliable and reproducible results for practical applications. ${ }^{[1]}$ Therefore, it has been reported in the literature aiming to eliminate beaded fibers and enhance the morphological uniformity of electrospun PS nanofibers. The results showed that formation of bead-free fibers is directly correlated to the stability of the "Taylor cone" and the steady-state condition of the jet, which could be visually examined through the electrospinning process. ${ }^{[12]}$ Moreover, it is well established that the electrospinning solution viscosity, surface tension, and electrical conductivity are the most influential factors that dictate the stability of the jet and thereby the morphology of the electrospun nanofibers. ${ }^{[13,14]}$ All the electrospinning solution variables could affect at least one of these three factors. Moreover, addition of different additives (e.g., auxiliary polymers, organic, and inorganic salts) is another option which could influence the three factors. ${ }^{[15,16]}$ With respect to the literature, most of the studies were concentrated on the fabrication of uniform and bead-free PS fibers by adjusting the type of solvent, electrospinning process parameters, solution parameters, and adding some additives (salts or surfactants) to the solution. ${ }^{[6,10,11]}$ However, none of these efforts have simultaneously investigated the effect of the parameters mentioned to achieve uniform and bead-free PS nanofibers with the minimum possible diameter.

Therefore, the main objective of this work was to optimize the electrospinning solution and process parameters to obtain morphologically fine PS nanofibers with the minimum possible diameter and narrow diameter distribution. For this aim, the effect of solvent type and electrolyte type (organic and inorganic salts) on the electrical conductivity, surface tension, and the viscosity of the electrospinning solution, as well as the morphology of the electrospun nanofibers, was assessed. The results showed that the beaded fibers disappeared once dimethylformamide (DMF) and sodium dodecylbenzenesulfonate (NaDDBS) were selected as favorable solvent and electrolyte, respectively. Modeling and optimization of the electrospinning process were undertaken using the statistical method of response surface methodology (RSM) to achieve PS nanofibers in the nanoscale range with the desired morphological characteristics. Overall, this study was conducted to provide a quantitative understanding of the influence of different solution and electrospinning variables and their interactions on the morphology and mean diameter of electrospun PS nanofibers.

\section{2 | EXPERIMENT}

\section{1 | Materials}

Linear PS $\left(M_{\mathrm{w}}=190,000 \mathrm{~g} / \mathrm{mol}\right)$, NaDDBS, zinc chloride $\left(\mathrm{ZnCl}_{2}\right), \mathrm{N}, \mathrm{N}$,-dimethylformamide (DMF), tetrahydrofuran (THF), N-methyl-2-pyrrolidone (NMP), toluene, and chloroform $(\mathrm{CF})$ were all purchased from Sigma-Aldrich (USA). All chemicals were used as received without further purification.

\section{2 $\quad$ Electrospinning process}

Organic solvents with different physicochemical properties were utilized to fabricate electrospun nanofibers. Some important properties of the solvents investigated in this work are listed in Table $1 .^{[17-19]}$

The PS solutions (20 wt \%) were prepared by dissolving a measured amount of PS pellets in different solvents (DMF, NMP, THF, CF, and Toluene). Moreover, to study the effect of different additives (salt and anionic surfactant) on the electrospinnability of the solutions, different concentrations of these additives were individually dissolved in the electrospinning solutions. 
TA B L E 1 Physical properties of solvents used in this research ${ }^{[16-18]}$

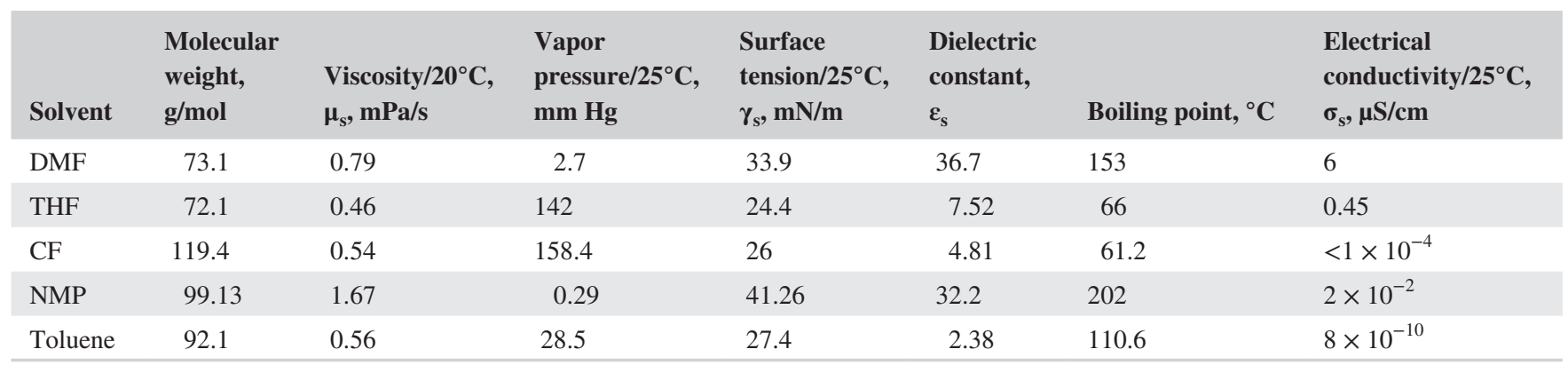

The as-prepared solutions were loaded into a 1-ml syringe supplied with a laser cut needle (gage 22, inner diameter of $0.4 \mathrm{~mm}$ ). The metallic needle was connected to a high-voltage supply, and a rotatable metal cylinder was grounded and used as collector for the electrospun nanofibers. Following preliminary experiments, the injection rate and the rotational speed of the collector were set at $0.3 \mathrm{ml} / \mathrm{hr}$ and $20 \mathrm{RPM}$, respectively, under room humidity and temperature conditions. The electrospun fibers were dried at room temperature for $24 \mathrm{hr}$ to remove the residual solvent.

\section{3 | Design of experiments}

Response surface methodology is a collection of empirical statistical modeling techniques useful for developing, improving, and optimizing the processes using quantitative data obtained from properly designed experiments to assess the individual behaviors and interactions of different independent variables. ${ }^{[3,20]}$ The main objectives of RSM are to find the coefficients of a mathematical model to verify the quantitative data obtained from experiments and determine the operational conditions for the system to achieve the optimum response (minimum, maximum, or a targeted value). ${ }^{[21]}$ In this regard, a Box-Behnken design (BBD) was employed to model the electrospinning process and study the effect of three independent parameters on the average nanofibers diameter, namely concentration of the electrospinning solution $\left(X_{1}\right.$, wt \%), applied voltage $\left(X_{2}, \mathrm{kV}\right)$, and the capillary tipcollector distance $\left(X_{3}, \mathrm{~cm}\right)$. The experimental range of each parameter, determined based on preliminary visual studies on the stability of electrospinning jet and evaluating the morphology of nanofibers with optical microscopy (OM; LeicaATC 2000, Germany), is summarized in Table 2. According to the BBD with three factors, 17 experimental runs were designed and the average nanofiber diameter was recorded as a response under the selected parameters. The data were analyzed by multiple regression analysis using the Design Expert software (trial version 8.0) to determine the optimum values of the selected variables for nanofibers with minimum diameter and generate response surfaces and contour plots.

\section{4 | Characterization}

The prepared solutions were characterized for their viscosity, electrical conductivity, and surface tension using a digital rotational viscometer (TQC DV1400, The Netherlands), a JENWAY conductivity meter (model 3540, Germany), and Du-Noüy ring method tensiometer (Krüss K-6, Germany), respectively. High-resolution scanning electron microscopy (SEM, LEO 1550 Gemini, Zeiss, Germany) was utilized to study the surface morphology of the nanofibrous samples. ImageJ Software (National Institute of Health, Bethesda, MD) was used to measure the average nanofiber diameter and nanofiber diameter distribution from SEM photomicrographs by 100 measurements of random fibers.

\section{3 | RESULTS AND DISCUSSION}

Among the various aforementioned parameters influencing the electrospinning process and the morphology of electrospun nanofibers, the solution and processing parameters are well known to be more determinative. Therefore, in this study, these two categories were optimized to fabricate PS nanofibers with uniform structure and as fine as possible diameter.

\section{1 $\quad$ Effect of solvent}

As the solution parameters can mutually influence each other, and independent assessments of them might not be comprehensive enough to provide a solid conclusion, through this research, the effect of different solution characteristics on the morphology and the average diameter of electrospun fibers were investigated. The rheological and electrical properties of polymer solutions (PS, $20 \mathrm{wt} \%$ ) with different solvents as well as the morphological results and mean diameter of the electrospun fibers obtained are summarized in Table 3. Moreover, the corresponding SEM images of the fibers (at low and high magnification) and their diameter distribution plots are shown in Figure 1. The results show that the mean fiber diameters of all the electrospun samples are in 
TA B L E 2 Independent variables used in experimental design and their levels in coded and actual values

\begin{tabular}{llllll} 
Parameters & Parameters description & Low level actual & High level actual & Low level coded & High level coded \\
\hline$X_{1}$ & $\begin{array}{l}\text { Solution concentration } \\
(\text { wt } \%)\end{array}$ & 15 & 21 & -1 & +1 \\
$X_{2}$ & $\begin{array}{l}\text { Applied voltage }(\mathrm{kV}) \\
X_{3}\end{array}$ & 14 & 20 & -1 & -1 \\
\hline \\
\hline
\end{tabular}

T A B L E 3 The characteristics of polystyrene (PS) solutions and the resulting electrospun PS fibers

\begin{tabular}{lllll}
$\begin{array}{l}\text { PS solu- } \\
\text { tions/20 wt } \% \\
\text { in }\end{array}$ & $\begin{array}{l}\text { Viscosity of } \\
\text { Solution }(\mathbf{m P a} / \mathbf{s})\end{array}$ & $\begin{array}{l}\text { Electrical conductivity of } \\
\text { solution }(\boldsymbol{\mu} \mathbf{S} / \mathbf{c m})\end{array}$ & $\begin{array}{l}\text { Surface tension of } \\
\text { solution }(\mathbf{m N} / \mathbf{m})\end{array}$ & $\begin{array}{l}\text { Nanofiber } \\
\text { morphology }\end{array}$ \\
\hline DMF & $206.7 \pm 4.7$ & $0.9 \pm 0.1$ & $34.9 \pm 0.4$ & $\begin{array}{l}\text { Mean nanofibers } \\
\text { diameter }(\mathbf{n m}) \\
\text { with beads }\end{array}$ \\
\hline CF & $246.2 \pm 4.1$ & a & $21.3 \pm 0.5$ & $\begin{array}{c}\text { Microfibers with } \\
\text { collapse beads }\end{array}$ \\
\hline THF & $272.5 \pm 4.3$ & a & $18.3 \pm 0.5$ & $\begin{array}{c}\text { Microfibers with } \\
\text { collapse beads }\end{array}$ \\
\hline Toluene & $242.5 \pm 2.5$ & a & $26.9 \pm 0.7$ & $\begin{array}{c}\text { Micro and nanofibers } \\
\text { with collapse beads }\end{array}$ \\
\hline NMP & $380 \pm 7.1$ & a & $36.7 \pm 0.6$ & $\begin{array}{c}\text { Very brittle fibers with } \\
\text { beads }\end{array}$ \\
\hline
\end{tabular}

${ }^{\mathrm{a}}$ Out of the detection limit of instrument.

micrometer range and have bead-on-string structures with different bead shapes. As the solution concentration and electrospinning parameters were all taken to be the same, the observed differences in the average fiber diameter, the shape, and the size of the beads could be ascribed to the rheological and electrical properties of the solutions (which arises from using different solvents).

The formation of microfibers with a bead-on-string structure for all the solutions with different solvents was due to insufficient stretching during the bending and whipping of the jet, which could be ascribed to the low electrical charge density and high surface tension of the solutions. The lower fiber diameters obtained from the solution prepared with DMF (as is summarized in Table 3) could be ascribed to the better electrical conductivity and lower viscosity of this solution $(0.9 \pm 0.1 \mu \mathrm{S} / \mathrm{cm}$ and $206.7 \pm 4.7 \mathrm{mPa} / \mathrm{s}$, respectively $)$ compared to the other solvents. The lower fiber diameters obtained from the solution prepared with NMP compared than those obtained from the solutions prepared with CF and THF might be attributed to the higher surface tension of this solution and the lower vapor pressure of this solvent. Both these factors could lead to slower jet solidification and thereby further jet elongation and thinner fibers.

Moreover, the SEM images at high magnification (Figure 1) show that the bead shapes varied drastically as a function of the solvent type. For the solvents with a relatively low boiling point (i.e., CF, THF, and Toluene) and ejecting the polymer solution from Taylor cone, a thin layer will be solidified on the surface of the jet in a very short time. Following evaporation of the residual solvent, vacuum hollow regions within the jet will be formed, which could lead to the collapse of the jet and formation of dimpled beads with irregular structures. ${ }^{[22]}$ In contrast, electrospun fibers with a round cross section and more spherical-like beads were formed by solvents with a relatively high boiling point (i.e., DMF and NMP), which could lead to a slower rate of solvent evaporation and more uniform solidification. The formation of spherical-like beads could also be attributed to the high surface tension of these solutions, which tends to minimize the jet surface and act against jet stretching and elongation. ${ }^{[10]}$ It was observed that the PS fibers electrospun with NMP solvent have low tendency to collect on the surface of the collector, which could be ascribed to the insufficient discharge of the jet and repulsion by the same charges on its surface. Overall, in accordance with the morphologies and the average diameters of electrospun nanofibers, DMF was found to be the most favorable solvent for electrospinning of PS and was chosen for further experiments.

Formation of bead-on-string structures indicated that the solution concentration was in the transition region. ${ }^{[12]}$ Although it is usually tacitly accepted that increasing the solution viscosity (which arises from an increase in the 

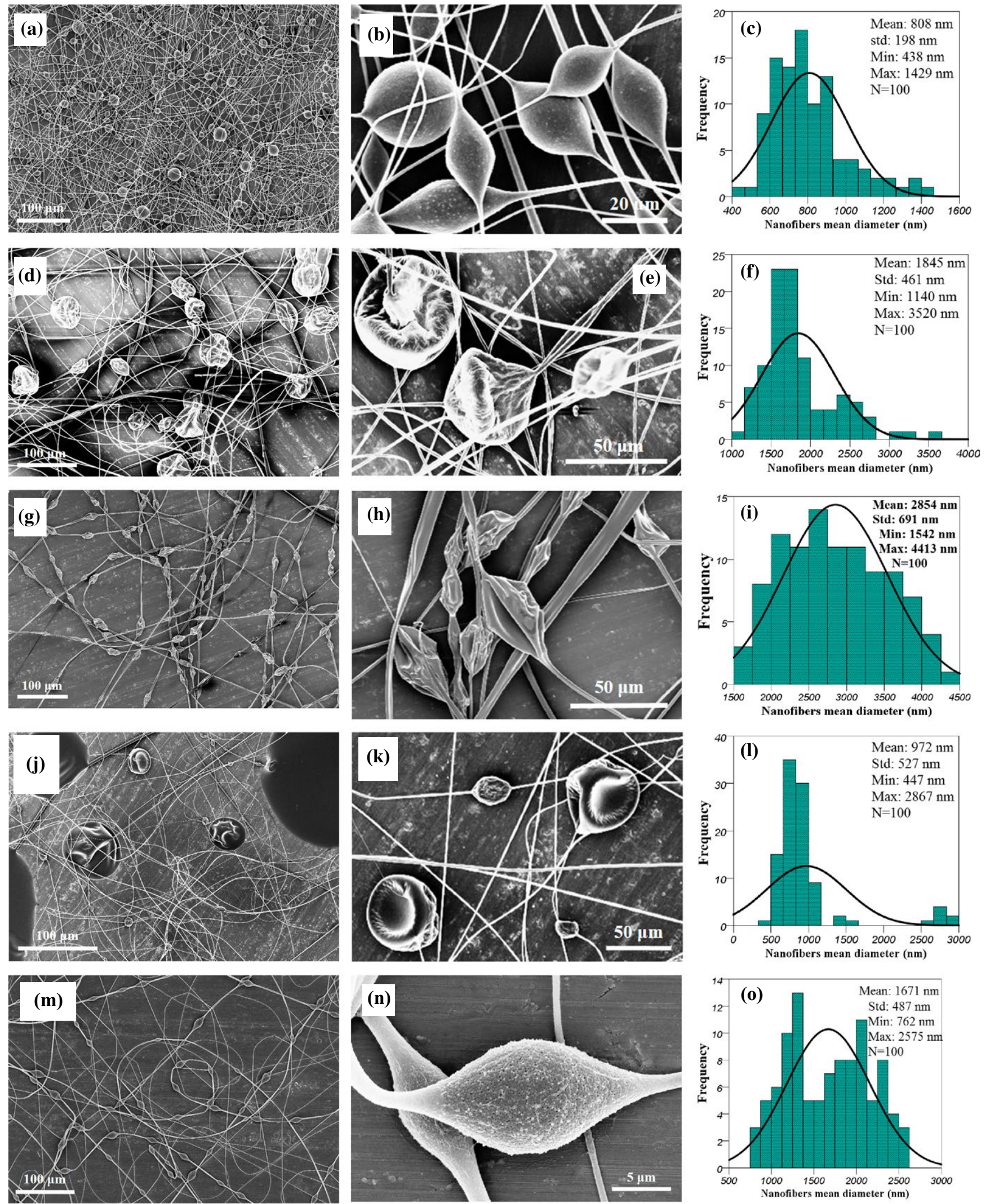

F I G URE 1 Scanning electron microscopy micrographs at low and high magnifications of polystyrene (PS) nanofibers electrospun with different solvent: dimethylformamide (a,b), chloroform (d,e), tetrahydrofurane $(\mathrm{g}, \mathrm{h})$, toluene $(\mathrm{j}, \mathrm{k})$, N-Methyl-2-pyrrolidone (m,n), and their diameters distributions: (c,f,i,l,o), respectively 
polymer concentration) could result in extensive polymerpolymer entanglements and thereby a quasi-stable jet, the diameter of electrospun fibers will also grow under the same processing conditions. ${ }^{[23]}$ Therefore, increasing the polymer concentration cannot solely be considered as an adequate strategy to fabricate uniform and bead-free fibers with as fine as possible diameter, and other solution parameters should be simultaneously taken into consideration. As the results indicated that the formation of bead-on-string structure is highly correlated to the electrical conductivity and surface tension of the electrospinning solution, the effect of some additives on these parameters, the morphology, and mean diameter of electrospun fibers were investigated.

\section{2 | Effect of organic and inorganic salts}

The rheological and electrical properties of PS solutions (20 wt\% in DMF) containing different concentrations of NaDDBS or $\mathrm{ZnCl}_{2}$ salts as well as the morphological results and mean diameter of the obtained electrospun fibers are summarized in Table 4. Moreover, the corresponding SEM images of the fibers and their diameter distribution plots are shown in Figure 2. The results showed that the viscosity of the electrospinning solutions was negligibly affected by addition of either $\mathrm{ZnCl}_{2}$ or NaDDBS. The negligible variation in the viscosity of solutions after adding the NaDDBS confirms that there is no surfactant/polymer interaction. ${ }^{[6]}$ Moreover, the slight reducing of the solution's viscosity after adding $\mathrm{ZnCl}_{2}$ could be ascribed to the decrease in DMF solvent power, which might result in less expanded polymer chains. ${ }^{[14,24]}$ Although the electrical conductivity is significantly increased by adding both salts, the improvement for the solutions containing $\mathrm{ZnCl}_{2}$ was more noticeable. As can be seen from Table 4, the surface tension decreased slightly by adding both organic and inorganic salts. These results are in accordance with literature reports, where a monotonic decrease in surface tension was observed with increasing organic and inorganic salt concentrations. ${ }^{[6,25,26]}$

It is clear from the images (Figure 2) that the beads disappeared and fibers with much thinner diameter and a round cross section had been fabricated, which confirms that the electrical conductivities are sufficient to overcome the surface tension and to form a stable jet. As the viscosity and surface tension of the electrospinning solutions are not significantly affected by the presence of the NaDDBS or $\mathrm{ZnCl}_{2}$ salts, it can be stated that the electrical conductivity plays a more crucial role in the formation of uniform fibers and can independently dictate the diameter of electrospun nanofibers. Increasing the solution conductivity could lead to higher charge density of the jet and therefore enhance the bending and whipping instabilities, resulting in the fading of the bead-on-string structures under the stronger stretching force. From Figure 2a and c, it is also evident that branch-like structures were achieved

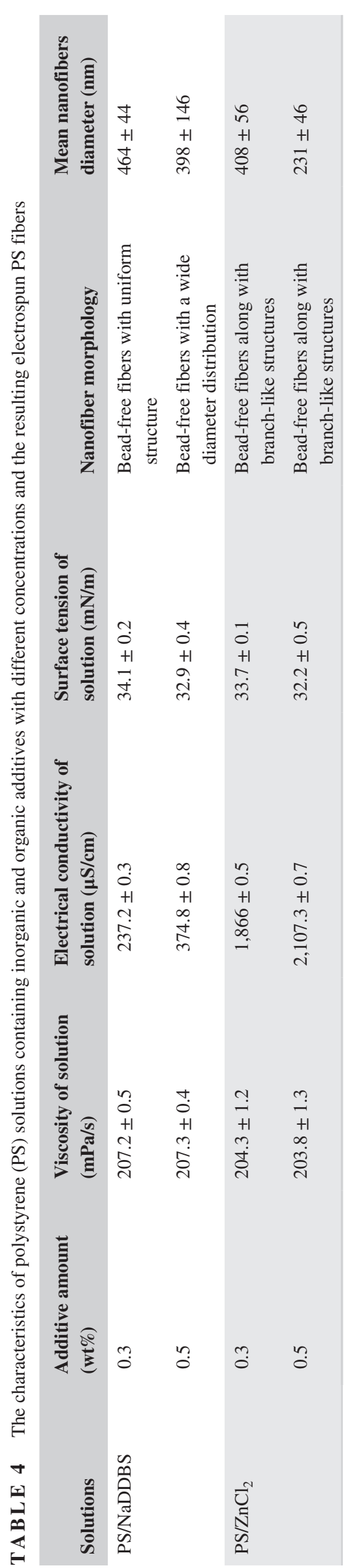



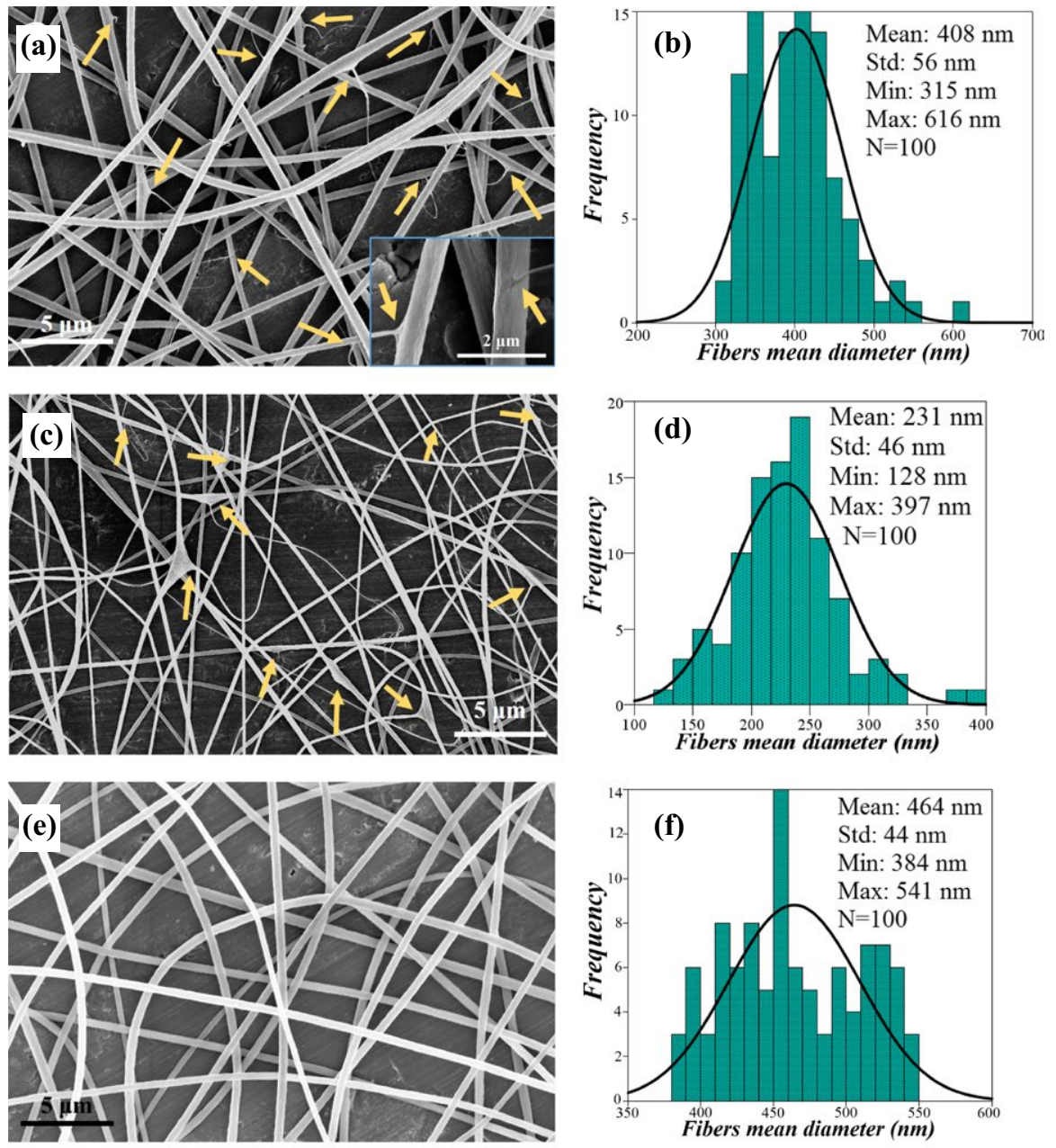

F I G URE 2 Scanning electron microscopy micrographs of polystyrene (PS) nanofibers (electrospun from $20 \mathrm{wt} \%$ PS solutions in dimethylformamide) and their diameter distributions with the following: $0.3 \mathrm{wt} \% \mathrm{ZnCl}_{2}$ (a,b), $0.5 \mathrm{wt} \% \mathrm{ZnCl}_{2}$ (c,d), $0.3 \mathrm{wt} \%$ sodium dodecylbenzenesulfonate (NaDDBS) (e,f), and $0.5 \mathrm{wt} \% \mathrm{NaDDBS}$ $(\mathrm{g}, \mathrm{h})$

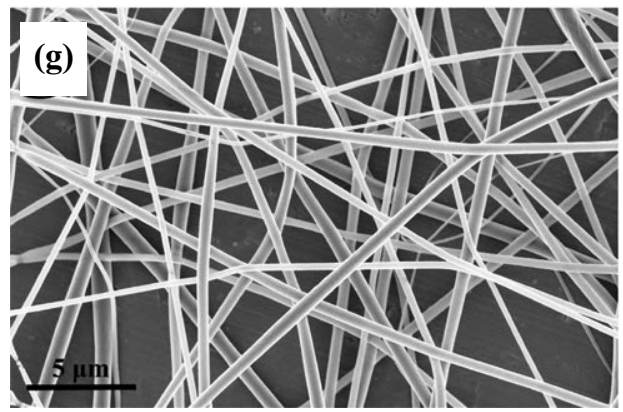

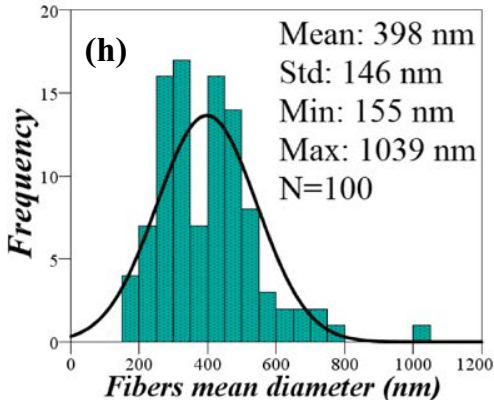

by adding $\mathrm{ZnCl}_{2}$ salt. The same fibers with branched structures are also observable in the literature that utilized other inorganic ionic salts (e.g., $\mathrm{LiCl}$ and $\mathrm{NaCl}$ ). ${ }^{[25,27,28]}$

Besides the higher ion density and lower surface tension of the solutions containing $\mathrm{ZnCl}_{2}$, the branched structure obtained (as are indicated with arrows in Figure 2a,c) might also be attributed to the smaller ionic radius of the dissociated salt, which could lead to higher mobility of the ions under an external electric field and thus splitting of the jet surface. ${ }^{[29]}$ While, more uniform fibers with a narrower diameter distribution were obtained when an organic ionic salt (NaDDBS, $0.3 \mathrm{wt} \%$ ) was added to the electrospinning solution. Although on increasing the NaDDBS concentration up to $0.5 \mathrm{wt} \%$, the mean diameter of fibers was slightly decreased (as is shown in Figure $2 \mathrm{~g}, \mathrm{~h}$ ), the diameter distribution and fiber uniformity deteriorated, which might be related to the visually observed jet splitting and instabilities through the electrospinning of this solution. Overall, it can be concluded that, although the presence of inorganic salt can obviously influence the diameter of electrospun nanofibers, it can also adversely affect the morphology and uniformity of the nanofibrous mat. Therefore, given the constructive effect of $0.3 \mathrm{wt} \%$ NaDDBS on the morphology and diameter of electrospun PS nanofibers, this inorganic salt was chosen for further experiments. 
TA B L E 5 Box-Behnken design matrix consisting of 17 experiments for three variables with actual values, along with the experimental and predicted values for polystyrene nanofibers diameters

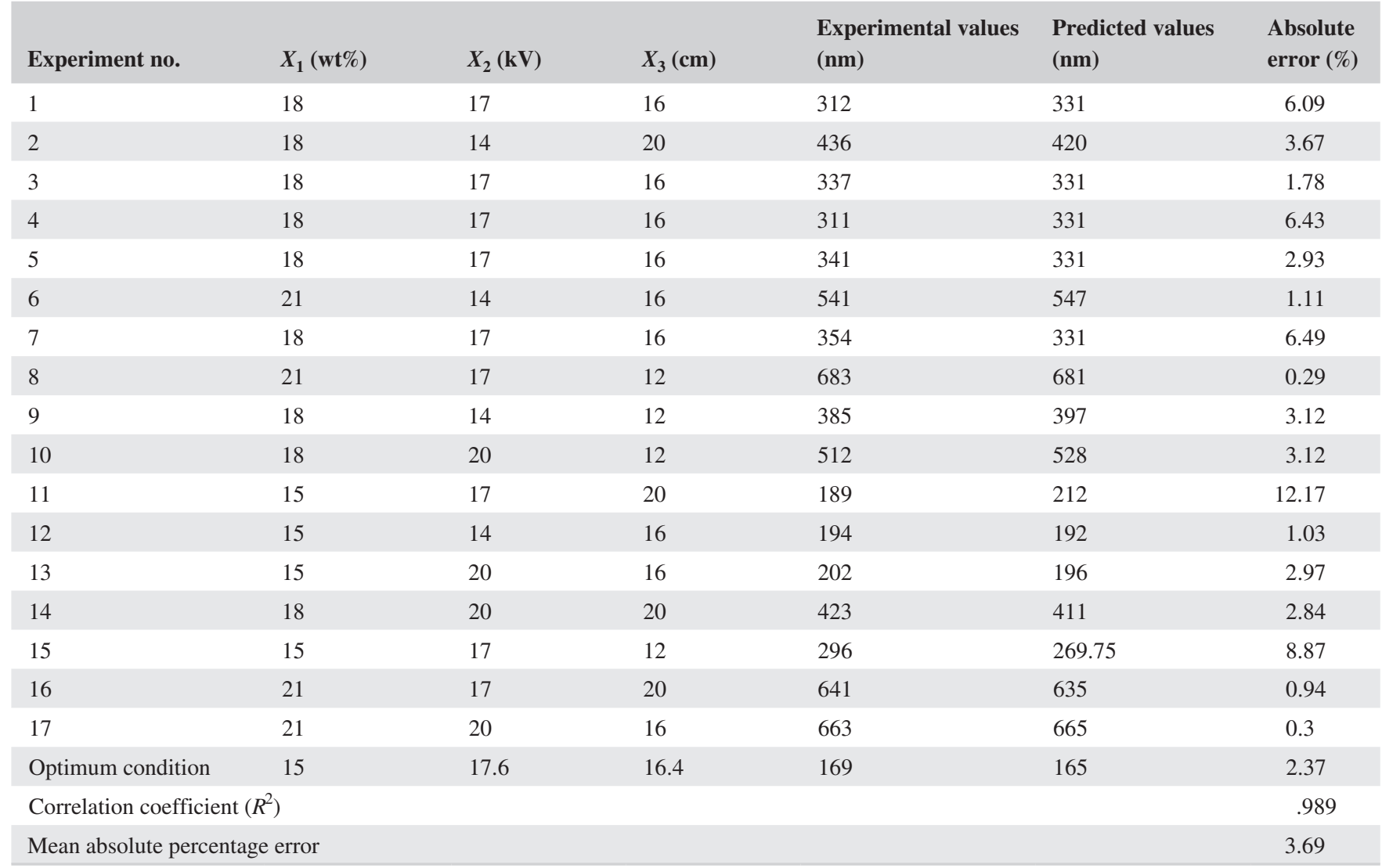

\begin{tabular}{|lccccc|}
\hline Source & SS & $d \boldsymbol{f}$ & MS & $\boldsymbol{F}$-value & Prob $>\boldsymbol{F}$ \\
\hline Model & $400,880.2$ & 9 & $44,542.25$ & 99.99061 & $<.0001$ \\
\hline$X_{1}$ & $339,076.1$ & 1 & $339,076.1$ & 761.1747 & $<.0001$ \\
\hline$X_{2}$ & 7,442 & 1 & 7,442 & 16.70617 & .0046 \\
\hline$X_{3}$ & $4,371.125$ & 1 & $4,371.125$ & 9.812515 & .0166 \\
\hline$X_{1} X_{2}$ & 3,249 & 1 & 3,249 & 7.293514 & .0306 \\
\hline$X_{1} X_{3}$ & $1,056.25$ & 1 & $1,056.25$ & 2.371122 & .1675 \\
\hline$X_{2} X_{3}$ & 4,900 & 1 & 4,900 & 10.99976 & .0128 \\
\hline$X_{1}^{2}$ & $7,121.118$ & 1 & $7,121.118$ & 15.98583 & .0052 \\
\hline$X_{2}^{2}$ & $3,271.645$ & 1 & $3,271.645$ & 7.344348 & .0302 \\
\hline$X_{3}^{2}$ & $27,031.64$ & 1 & $27,031.64$ & 60.68196 & .0001 \\
\hline Residual & $3,118.25$ & 7 & 445.4643 & & \\
\hline Lack of fit & $1,692.25$ & 3 & 564.0833 & 1.582281 & .2703 \\
\hline Pure error & 1,426 & 4 & 356.5 & & \\
\hline Total & $403,998.5$ & 16 & & & \\
\hline
\end{tabular}

TABLE 6 Analysis of variance (ANOVA) for the parameters of response surface methodology fitted to a quadratic polynomial equation

SS, sum of squares; $d f$, degree of freedom; MS, mean square; $X_{1}$, solution concentration; $X_{2}$, applied voltage; $X_{3}$, capillary tip-collector distance. $R^{2}=.9897, R_{\text {adj }}^{2}=.9793$.

\section{3 | Effect of electrospinning parameters}

As there are multiple electrospinning variables which have potential to influence the final characteristics of the nanofibers, the concurrent evaluation of all these parameters is almost impossible. ${ }^{[30]}$ In this context, it should be mentioned that the impact and the weight of these variables are not identical. Therefore, to effectively model and optimize 
the electrospinning process, the influence of the most significant parameters was investigated as model variables and all others were kept constant. According to the preliminary experiments, the solution concentration, capillary tip-collector distance, and applied voltage were recognized and selected as the factors that can highly impact the morphology and diameter of the electrospun nanofibers. The mean nanofiber diameter was taken as the response to the design experiments. RSM based on BBD was applied as an effective multivariate statistical method to model the electrospinning process, investigate the effect of each variable and their interactions, and obtain PS nanofibers with the minimum possible diameter. The experimental range of the variables was determined in accordance with the preliminary experiments (as is summarized in Table 2). The designed experiments (Table 5) were conducted, and a second-order regression mathematical
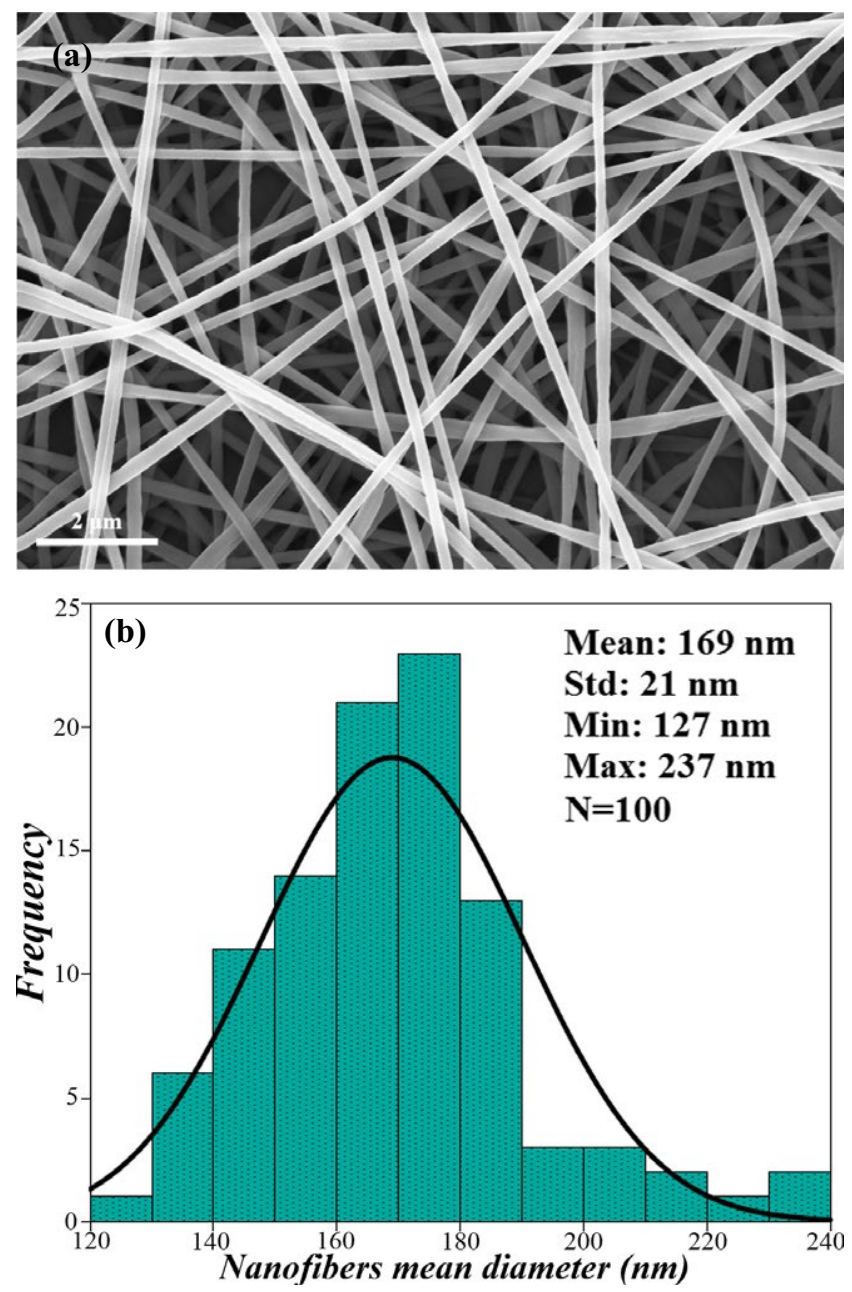

F I G URE 3 Scanning electron microscopy micrograph of polystyrene (PS) nanofibers electrospun under the optimum condition: PS solution concentration of $15 \mathrm{wt} \%$ in dimethylformamide solvent, sodium dodecylbenzenesulfonate concentration of $0.3 \mathrm{wt} \%$, applied voltage of $17.6 \mathrm{kV}$, and capillary tip-collector distance of $16.4 \mathrm{~cm}$ (a), and its diameter distribution (b) model was developed to predict the electrospun PS nanofibers, the impact of each parameter, and their interactions.

An analysis of variance (ANOVA) of the experimental results was conducted to identify the statistical significance of each studied variables and is shown in Table 6 . The response surface model had a high correlation coefficient $\left(R^{2}=.989\right)$ and nonsignificant lack of fit (0.2703), which indicated that the proposed model could properly explain the experimental data. The coefficients of the modified model with their respective $p$-values were calculated in accordance with a multiple regression analysis. The modified regression equation (modified model) involving the statistically significant terms (terms with $p$-value <.05) was obtained and is presented in Equation (1):

$$
\begin{aligned}
\eta= & 2849.68056-149.70833 \times X_{1} \\
& -105.47222 \times X_{2}-116.51042 \times X_{3} \\
& +3.16667 \times X_{1} X_{2}-2.91667 \times X_{2} X_{3} \\
& +4.56944 \times X_{1}^{2}+3.09722 \times X_{2}^{2}+5.00781 \times X_{3}^{2}
\end{aligned}
$$

where $\eta$ is the average PS nanofibers diameter $(\mathrm{nm}), X_{1}$ is the solution concentration (wt\%), $X_{2}$ is the applied voltage $(\mathrm{kV})$, and $X_{3}$ is the capillary tip-collector distance $(\mathrm{cm})$.

According to the modified model, the optimum conditions for producing PS nanofibers with the minimum possible diameter were specified as follows: PS solution concentration of $15 \mathrm{wt} \%$, applied voltage of $17.6 \mathrm{kV}$, and capillary tip-collector distance of $16.4 \mathrm{~cm}$. The model anticipates that nanofibers with mean diameter of $165 \mathrm{~nm}$ would be fabricated under the stated conditions. In order to assess the validity and reliability of the modified model, the electrospinning process under the optimized condition was performed and the experimental value of the mean nanofibers diameter was compared to that of predicted from the modified model (as is summarized in Table 5). An SEM image of the optimized nanofibers and their diameter distribution are presented in Figure 3.

From the SEM image, it can be seen that uniform nanofibers with a cylindrical shape, smooth surface, and average diameter of $169 \pm 21 \mathrm{~nm}$ were successfully produced. The very close correlation between the experimental values and the estimated responses clearly verifies the accuracy and precision of the proposed model. The optimized model not only explains the impact and the weight of each parameter and their interactions, but could also serve as a convenient model for further studying the electrospinning process with the same PS solutions.

\subsection{Evaluation of the effect of independent parameters and their interactions}

The perturbation plot of the effects of independent parameters and the contour plots of their interactions on the mean diameter of the nanofibers are shown in Figure 4. From Figure 4a, it can be observed that although decreasing both the solution 


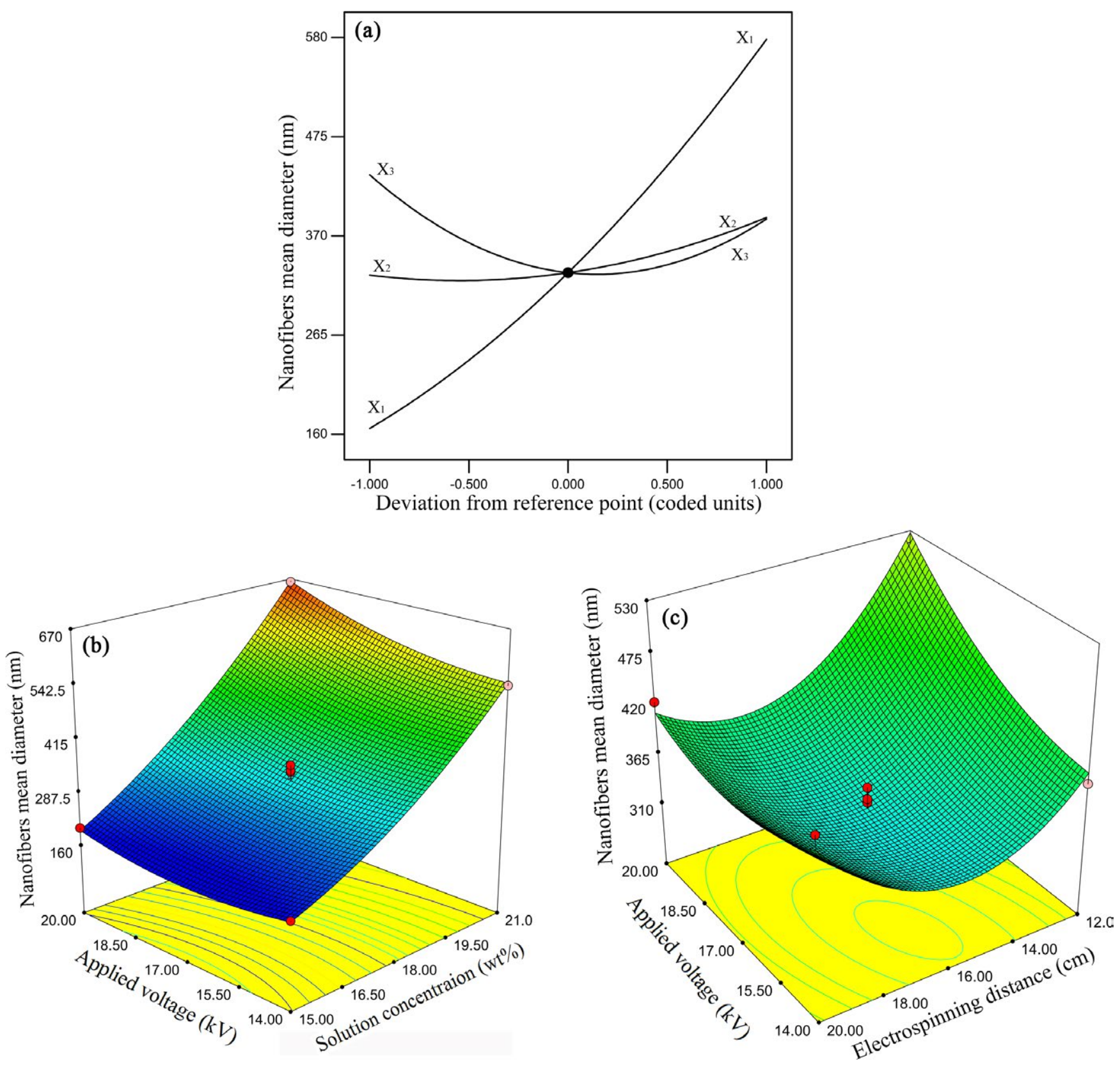

F I G U RE 4 Perturbation plot of the parameter effects on the polystyrene (PS) nanofiber diameter (a), response surfaces for mean PS nanofiber diameter in terms of solution concentration-applied voltage interaction (b), and applied voltage-electrospinning distance interaction (c). The third factors (i.e., $X_{3}$ and $X_{1}$ in b and c, respectively) are considered constant at their central values. $X_{1}$, solution concentration; $X_{2}$, applied voltage; $X_{3}$, capillary tip-collector distance $s$

concentration $\left(X_{1}\right)$ and the applied voltage $\left(X_{2}\right)$ could result in electrospun PS nanofibers with relatively small diameters, this downward trend with solution concentration was much more drastic and significant. It is usually accepted that increasing the polymer concentration would lead to further polymer chain entanglements and higher viscoelastic forces, which could resist the electrostatic stretching forces through the electrospinning process. ${ }^{[31]}$ Similar results have been reported in the literature. ${ }^{[3,32]}$ Whereas, in the case of the effect of applied voltage on the nanofiber's diameter, there is no definitive formulation and several contradictory results have been reported. ${ }^{[30]}$ Increasing the applied voltage, on the one hand, leads to decreasing the flight time of the jet and, on the other hand, results in a stronger electrostatic stretching force, which tends to increase and decrease the nanofiber diameter, respectively. Moreover, it has also been claimed that increasing the applied voltage may cause a higher quantity of polymer solution to extrude out of the jet and thus form of thicker electrospun nanofibers. ${ }^{[30]}$ The present study indicates that the resultant effects of the applied voltage on the mean diameter of electrospun nanofibers are slow and incremental. Moreover, the results showed that the effect of 
递

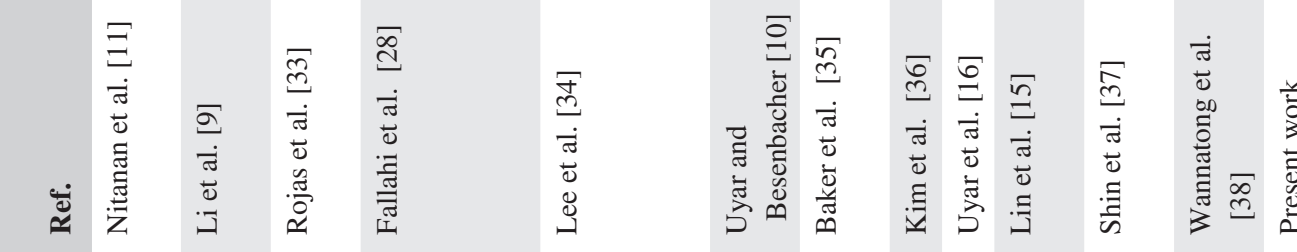

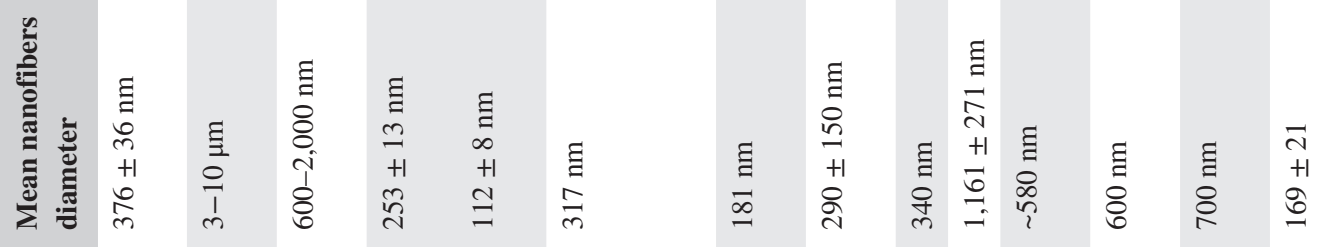

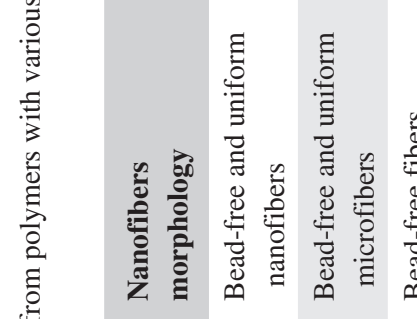

$\frac{2}{2}$

矛

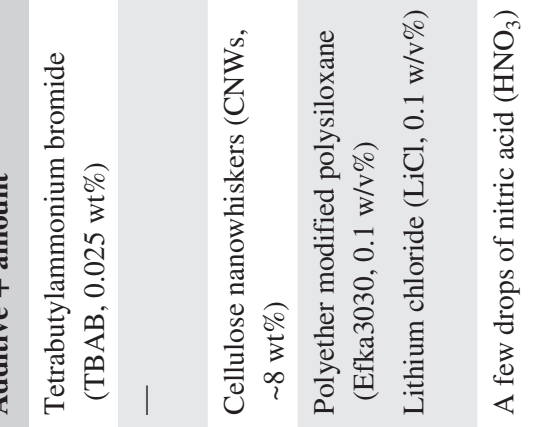

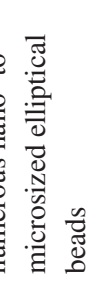

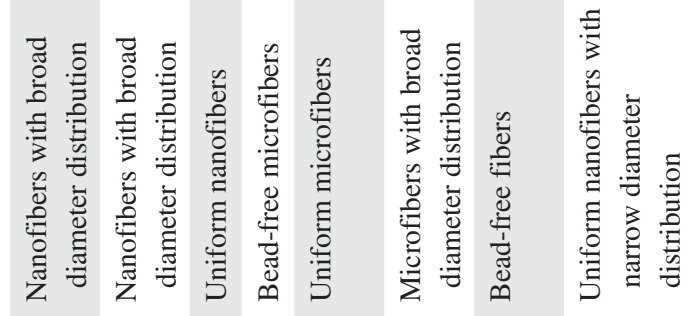

0
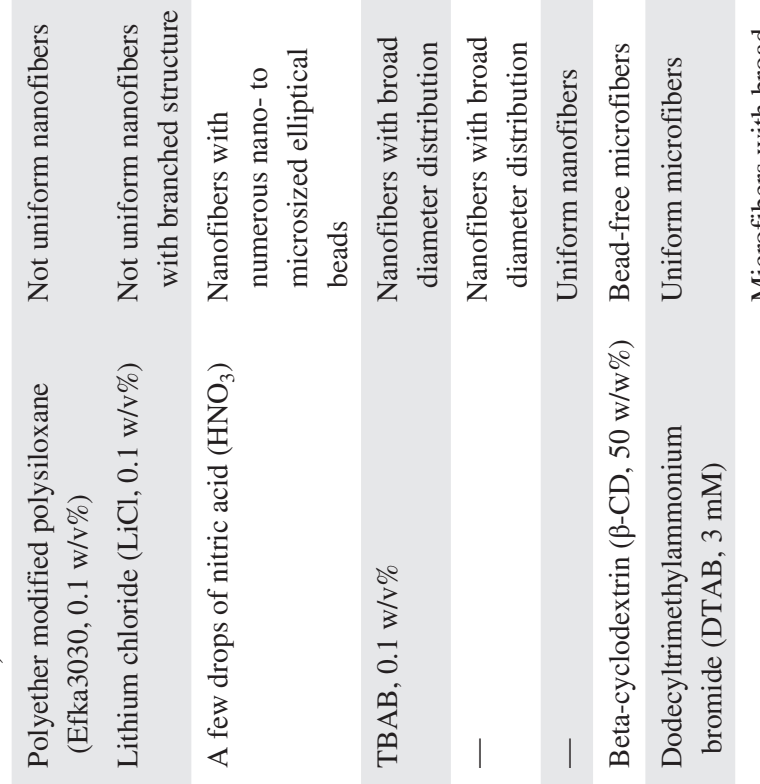

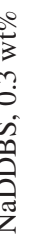

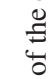

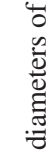

ฐี

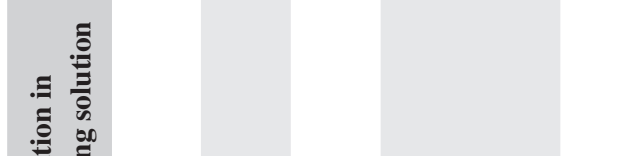

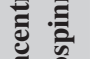

这

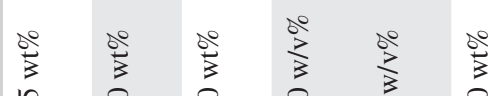

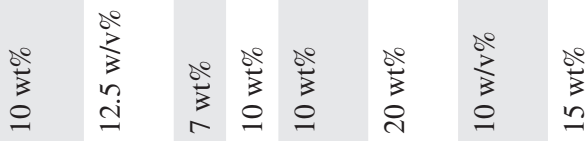

होin

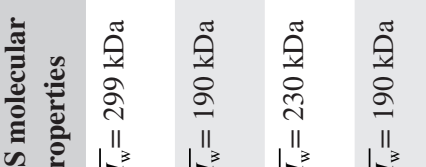

$\frac{\tilde{9}}{8}$

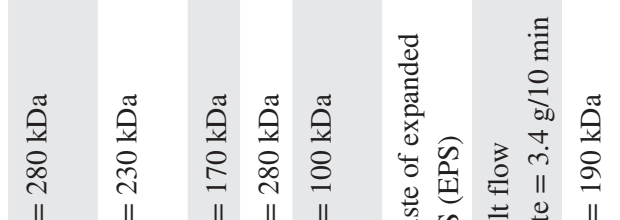

竞

过

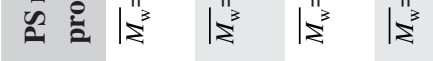
$\left.\right|^{11}$

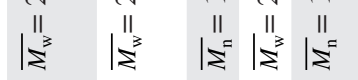

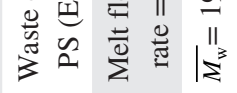

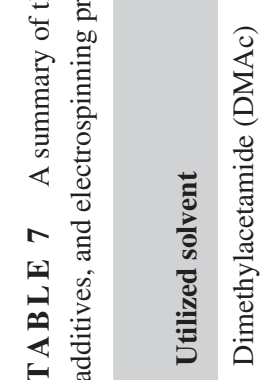

言崖言

言

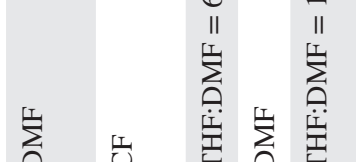

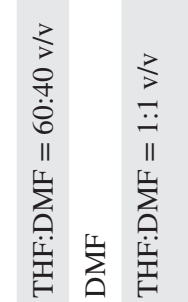


the capillary tip-collector distance $\left(X_{3}\right)$ on the mean diameter of electrospun PS nanofibers is not always consistent, so that an extreme value could be identified through the experimental range. 3D surface plots of the simultaneous effects of solution concentration-applied voltage and capillary tip-collector distance-applied voltage on the mean diameter of the nanofibers are illustrated in Figure 4b,c, respectively.

For both of the 3D surface plots presented, the simultaneous effect of two factors on the response variable $(\eta)$ was investigated and the third parameter (i.e., $X_{3}$ and $X_{1}$ in Figure $4 b, c$, respectively) was kept constant at its respective zero level (central value). From the plots, it is clear that the mean diameter of PS nanofibers at high level of solution concentration (Figure 4b) and low level of capillary tip-collector distance (Figure 4c) was more significantly correlated to the applied voltage variations. Figure $4 \mathrm{~b}$ suggests that simultaneously increasing the polymer concentration and applied voltage could result in PS nanofibers with thicker diameters. Overall, it could be stated that the impact and the weight of the solution concentration are considerably higher than those of the two other studied parameters, and to achieve PS nanofibers with superfine diameter, this parameter should be carefully taken into consideration.

\subsection{Comparison with the other reports}

A summary comparison of the existing studies on the electrospinning process to fabricate PS nanofibers with desired morphological characteristics is shown in Table 7. ${ }^{[16,33-38]}$ Considering the electrospun nanofiber morphologies as well as their mean diameters, the optimized nanofibers fabricated in this study have some of the finest diameters along with a smooth surface and remarkable diameter uniformity.

Besides the solution and electrospinning process parameters, the presence and the type of additives (electrolytes) are the most important factors which dictate the morphology and mean diameter of PS nanofibers. Given the advantages of PS nanofibers, this optimized structure is a promising material for a wide range of potential applications from biosensing ${ }^{[39]}$ to oil spill remediation. ${ }^{[34]}$

\section{4 | CONCLUSION}

We present a systematic study of the influence of different solution and electrospinning variables on the morphology and mean diameter of electrospun PS nanofibers to obtain nanofibers with the minimum possible diameter. The effects of five solvents (i.e., DMF, THF, CF, NMP, and Toluene) on the viscosity, electrical conductivity, surface tension, and the morphology of the electrospun nanofibers were assessed. Although none of these solutions provided bead-free fibers, DMF was found to be the more promising solvent, owing to its better electrical conductivity and lower viscosity $(0.9 \pm 0.1 \mu \mathrm{S} / \mathrm{cm}$ and $206.7 \pm 4.7 \mathrm{mPa} / \mathrm{s}$, respectively), and was chosen for further experiments. Moreover, the SEM results indicated that even the shape of the beads is dependent upon the type of solvents, so that more spherical-like beads could be formed from solvents with lower volatility and higher surface tension. The influence of different ionic additives $\left(\mathrm{ZnCl}_{2}\right.$ and NaDDBS as inorganic and organic salts, respectively) on the physicochemical properties of the solutions and the morphology of the electrospun nanofibers were also assessed. Contrary to expectations, the results showed that, although the presence of $\mathrm{ZnCl}_{2}$ strongly reduced the mean diameter and formed bead-free nanofibers, the morphology deteriorated and branch-like structures appeared on the surface of nanofibers. However, by adding NaDDBS, nanofibers with the desired uniformity were formed. Finally, to model the electrospinning process of PS and achieve nanofibers with the minimum possible diameter, RSM was employed as an effective statistical method. The nanofibers with the finest diameter of $169 \pm 21 \mathrm{~nm}$ were obtained under the optimal conditions suggested by the model, which was in close agreement with the predicted value (the error percentage of $2.37 \%$ ). Compared to previous research on electrospun PS nanofibers, the optimized nanofibers fabricated in this work have some of the finest diameters together with a smooth surface and remarkable diameter uniformity.

\section{ACKNOWLEDGMENTS}

BR would like to gratefully acknowledge the Ministry of Science, Research and Technology of Iran for financial support that allowed him to work at Linköping University.

\section{ORCID}

Babak Rezaei (D) http://orcid.org/0000-0002-8177-5363

Ahmad Mousavi Shoushtari

http://orcid.org/0000-0001-8138-5560

\section{REFERENCES}

[1] N. Bhardwaj, S. C. Kundu, Biotechnol. Adv. 2010, 28, 325.

[2] B. Rezaei, M. Ghani, A. M. Shoushtari, M. Rabiee, Biosens. Bioelectron. 2016, 78, 513.

[3] B. Rezaei, M. Askari, A. M. Shoushtari, R. A. M. Malek, J. Therm. Anal. Calorim. 2014, 118, 1619.

[4] J.-P. Chen, K. H. Ho, Y. P. Chiang, K. W. Wu, J. Membr. Sci. 2009, 340, 9 .

[5] H. An, C. Shin, G. G. Chase, J. Membr. Sci. 2006, 283, 84.

[6] T. Lin, H. Wang, H. Wang, X. Wang, Nanotechnology 2004, 15 , 1375 . 
[7] N. Amiraliyan, M. Nouri, M. H. Kish, Fiber Polym. 2009, 10, 167.

[8] S. H. Tan, R. Inai, M. Kotaki, S. Ramakrishna, Polymer 2005, 46, 6128.

[9] X. Li, C. Wang, Y. Yang, X. Wang, M. Zhu, B. S. Hsiao, Appl. Mater. Interfaces 2014, 6, 2423.

[10] T. Uyar, F. Besenbacher, Polymer 2008, 49, 5336.

[11] T. Nitanan, P. Opanasopit, P. Akkaramongkolporn, T. Rojanarata, T. Ngawhirunpat, P. Supaphol, Korean J. Chem. Eng. 2012, 29, 173.

[12] G. Eda, J. Liu, S. Shivkumar, Eur. Polym. J. 2007, 43, 1154.

[13] W. Klairutsamee, P. Supaphol, I. Jangchud, J. Appl. Polym. Sci. 2015, 132, 42716.

[14] W. Ding, S. Wei, J. Zhu, X. Chen, D. Rutman, Z. Guo, Macromol. Mater. Eng. 2010, 295, 958.

[15] J. Lin, B. Ding, J. Yu, ACS Appl. Mater. Interfaces 2010, 2, 521.

[16] T. Uyar, R. Havelund, J. Hacaloglu, X. Zhou, F. Besenbacher, P. Kingshott, Nanotechnology 2009, 20, 125605.

[17] I. Smallwood, Handbook of Organic Solvent Properties, Arnold/ Halsted Press, London 1996.

[18] K. Nasouri, A. M. Shoushtari, M. R. M. Mojtahedi, Adv. Polym. Technol. 2015, 34, 1098.

[19] S. Megelski, J. S. Stephens, D. B. Chase, J. F. Rabolt, Macromolecules 2002, 35, 8456.

[20] Q. Wang, X. R. Fan, Z. Z. Hua, J. Chen, Biochem. Eng. J. 2007, 34, 107.

[21] M. Ghani, B. Rezaei, A. L. Ghare Aghaji, M. Arami, Adv. Polym. Technol. 2016, 35, 428.

[22] B. Rezaei, M. Ghani, M. Askari, A. M. Shoushtari, R. M. A. Malek, Adv. Polym. Technol. 2016, 35, 21534.

[23] C. Shin, G. G. Chase, Polym. Bull. 2005, 55, 209.

[24] X. H. Qin, E. L. Yang, N. Li, S. Y. Wang, J. Appl. Polym. Sci. 2007, 103, 3865.

[25] K. P. Matabola, R. M. Moutloali, J. Mater. Sci. 2013, 48, 5475.

[26] K. Arayanarakul, N. Choktaweesap, D. Aht-ong, C. Meechaisue, P. Supaphol, Macromol. Mater. Eng. 2006, 291, 581.
[27] X. Lu, J. Zhou, Y. Zhao, Y. Qiu, J. Li, Chem. Mater. 2008, 20, 3420.

[28] D. Fallahi, M. Rafizadeh, N. Mohammadi, B. Vahidi, E-Polymers 2008, 8,1 .

[29] X. Zong, K. Kim, D. Fang, S. Ran, B. S. Hsiao, B. Chu, Polymer 2002, 43, 4403 .

[30] M. Askari, B. Rezaei, A. M. Shoushtari, P. Noorpanah, M. Abdouss, M. Ghani, Can. J. Chem. Eng. 2014, 92, 1008.

[31] M. Ziabari, V. Mottaghitalab, A. K. Haghi, Korean J. Chem. Eng. 2010, 27, 340 .

[32] O. S. Yördem, M. Papila, Y. Z. Menceloğlu, Mater. Des. 2008, 29, 34.

[33] O. J. Rojas, G. A. Montero, Y. Habibi, J. Appl. Polym. Sci. 2009, 113, 927.

[34] M. W. Lee, S. An, S. S. Latthe, C. Lee, S. Hong, S. S. Yoon, ACS Appl. Mater. Interfaces 2013, 5, 10597.

[35] S. C. Baker, N. Atkin, P. A. Gunning, N. Granville, K. Wilson, D. Wilson, J. Southgate, Biomaterials 2006, 27, 3136.

[36] G.-T. Kim, Y. J. Hwang, Y. C. Ahn, H. S. Shin, J. K. Lee, C. M. Sung, Korean J. Chem. Eng. 2005, 22, 147.

[37] C. Shin, G. G. Chase, D. H. Reneker, Colloids Surf. A Physicochem. Eng. Asp. 2005, 262, 211.

[38] L. Wannatong, A. Sirivat, P. Supaphol, Polym. Int. 2004, 53, 1851.

[39] V. Kunduru, M. Bothara, J. Grosch, S. Sengupta, P. K. Patra, S. Prasad, Nanomed. Nanotechnol. Biol. Med. 2010, 6, 642.

How to cite this article: Rezaei B, Shoushtari AM, Rabiee M, Uzun L, Turner APF, Cheung Mak W. Multifactorial modeling and optimization of solution and electrospinning parameters to generate superfine polystyrene nanofibers. Adv Polym Technol. 2018;00:1-13. https://doi.org/10.1002/adv.21947 